\title{
A Review on the Removal of Dye, Pesticide and Pathogens from Waste Water Using Quantum Dots
}

\author{
Kalaiselvi Velayutham Anand, Sandy Subala Anand, Sumathi K. Sripathi, \\ and Antony Lucia Merin Stanish
}

\section{ABSTRACT}

The industrial pollutants in water bodies tend to unsuitable for living organisms and irrigation uses. Water contamination is exaggerating at regular pace and the universe is holding carcinogenic agents. Therefore, there is a necessity of immediate action to generate a potential and efficient technology for water management. By means of this, quantum dots (QDs) have emerged as an effective probe for the removal process. This review discusses the methods for removing and degrading the coloured components, pesticides, pathogens from waste water and contaminants removal ability of QDs.

Keywords: Carbon Quantum Dots, Chemiluminescence, Methylene Blue, Photo catalyst.
Published Online: September 29, 2020

ISSN: $2684-4478$

DOI: $10.24018 /$ ejchem.2020.1.5.14

Kalaiselvi Velayutham Anand*

DMI St. Eugene University, Lusaka, Zambia.

(e-mail: anandvelu4@gmail.com) Sandy Subala Anand

DMI St. Eugene University, Lusaka, Zambia.

(e-mail: sibisandy@ gmail.com)

Sumathi K. Sripathi

DMI St. Eugene University, Lusaka, Zambia.

(e-mail: sumathiksripathi@ gmail.com)

Antony Lucia Merin Stanish

DMI St. Eugene University, Lusaka,

Zambia.

(e-mail: luciamerin31@gmail.com)

*Corresponding Author

\section{INTRODUCTION}

Water management is a universal question nowadays with the growing demand of freshwater caused by increasing population, domestic usage, and emerging industrial needs. Waste water treatment is the basic and fundamental process to stay clear from polluted water for human consumption. The water bodies are contaminated due to the pollution from discarded items such as coloured materials, pesticides, herbicides, pathogens and heavy metals due to human utilization. Therefore, efficient technology and methods have been implemented for the effective removal of pollutants from sewer water. Several technology and procedures are being adopted for removing and degrading the contaminants during the purification process[1].Various processes were identified for the development of water purification technologies including coagulation, sedimentation, filtration, chemical purification, ion exchange, reverse osmosis, etc. Even though few water treatment methods are established, the efficiency of the nanomaterials in the water purification could be improved by developing novel filtration membrane, high surface area adsorbents, and other processes [2].The objective of all these processes is the removal of contaminants from water and to provide clean water. Since nanotechnology emerges, it has a vital role in waste water treatment due to the ability of biological tomography, particle sensing and heavy metal detection. Different form of water treatment procedures is generated by several research groups around the world by making various nanomaterials into the water treatment process [3]. Numerous nanomaterials have been attempted to validate the removal efficiency and to reduce the cost of the overall process. Even though the commercially available nanomaterial has been utilized for water purification process, several research groups are in the process of synthesizing or developing various nanomaterial systems to improve the efficiency by obtaining various morphologies and different hybrid system. The use of semiconductor nanocrystal or QDs is beneficial over other materials in terms of luminescence, photostability and photocatalyst. The purification performance may vary with parameters such as $\mathrm{pH}$, doping agent and the QDs used for the purification process. The fresh water polluted with coloured impurities from industries become more likely to health issues such as hematolysis, high blood pressure, and respiratory disorder in living organisms [4]. The coloured impurities or dyes are classified into anionic, cationic and nonionic. The dyes used in fabric industries discharged into fresh water bodies leads to contamination of water. These dyes dissolved in water 
and produce intense colours with acidic properties. These colourants consume the oxygen in the water [5].

Dyes discharged into water bodies from various manufacturing unit, such as leather, food industry, paper, cosmetics, pharma companies etc. The textile and dyeing industries use large quantities of dyes and expel these colourants into ecosystem as water pollutants. These toxicants are unsafe to living organisms, thus it should be removed from the water resources in order to make it suitable for consumption. These dyes are non-biodegradable and stable to light. They are resistive towards aerobic digestion and meant for the hard groups to be eliminated from the industrial wastewater [6].

Microorganisms and pesticides pose a threat to the universe due to their bio accumulative feature which has negative impact on the environment and human health [7]. By and large, the hazardous chemical detection was tedious and expensive process. However, graphene nano materials in bio sensing techniques plays an important role due to the unique and excellent electronic and optical properties [8].

Photocatalysis is an essential factor for large number of ecological and energy concern at the moment. Herein, semiconductors generally play this role due to their band gaps in the process of absorbing light over a wide range of spectra. This is achieved by the semiconductor in which the absorbed energy should be equal to or larger than the band gap in order to form electron hole pair. Nanoscience research has a strong influence on the development of novel and more influential catalysts through the pattern and properties through with energy gap, composition and modification $[9,10]$. Since nanotechnology emerges, QDs are promising materials for photo-catalysis, ions sensing, biological imaging and heavy metal detection.

\section{QUANTUM DOTS}

Quantum Dots (QDs) are zero dimensional semiconductor nano crystals which is responsible for the light absorption and emission. The optical properties are further enhanced by fencing the core material with semiconductor material which limits the chemical attack. Since the last two decades these materials gain much attention due to the optoelectronic and optical properties [11,12]. A substantial quantity of research is focusing on utilizing the optical properties of QDs in several devices. The distinct feature of QDs is quantum confinement which is observable when the QDs size is small [13]. The important characteristic to be considered in QDs is doping when it is used for technical purposes such as optoelectronic, magnetic and biological applications [14], [15]. The relative position and amount of the dopant decides the optoelectronic and optical properties of QDs [16], [17]. The surface to volume ratio has significant effects on the optical properties [18].

The high cost of inorganic QDs is replaced by cost effective graphene quantum dots (GQD) and carbon quantum dots (CQD). Additionally, the excellent solubility, atoxic, good surface grating and stable photoluminescence making them as a potential candidate in technological applications. CQDs are very small carbon nanoparticles with high quality photoluminescence, greater degree of stability and conductivity. Owing to its unique properties it has received a wide attention among the researchers [19]-[21]. In general, CQDs can be synthesized by conventional methods such as electrochemical oxidation, laser ablation, hydrothermal/solvothermal treatment, microwave irradiation and arc discharge [22], [23].

The quantum confinement and edge effects the GQDs causes it as a potential candidate in several research fields [24]. Besides, they have unique properties such as low toxicity, greater solubility, chemical stability, photoluminescence stability and excellent surface grating etc. [25], [26]. Further, graphene QDs with intrinsic structures exhibit different selective quenching phenomena there by, making it possible to detect various ions and chemical groups. This concept denotes the fact that within the blue green region of the spectrum a striking energy band of PL characteristic of graphene QD is observed [27].

\section{DYE REMOVAL}

The fresh water resources are contaminated with the dye stuffs released from the textile, leather and cosmetic industries, results the reducibility of sunlight and prevent the photosynthesis of hydrophytes [28]. Photo catalytic approach is the long familiar method to get rid of these dyes. For example, the $\mathrm{N}$ doped CQDs bear distinct properties such as carbon work function reduction, enhancing photoluminescence emission ability and desirable electronic and optical properties. In this sense, $\mathrm{N}$ doped Carbon $\mathrm{QDs} / \mathrm{TiO}_{2}$ composites, photodegrade $86.9 \%$ of Methylene Blue (MB) compare with pristine $\mathrm{TiO}_{2}$, it was $53.8 \%$ due to the high charge separation in $\mathrm{CQDs} / \mathrm{TiO}_{2}$. It follows dye photosensitization mechanism [29]. In the degradation of fast Green $(\mathrm{FG})$, the cationic dye in the presence of bimetallic Fe@Ag CQDs synthesized by reduction method shows a band gap of $1.9 \mathrm{cV}$. The low saturation magnetization value shows that their ferromagnetic behavior does not depend upon the non-ferromagnetic Ag. The free radicals produced by Ag attack the adsorbed dye molecules causes the degradation of FG follows pseudo first order kinetics [30]. Whereas the $\mathrm{Fe}^{3+}$ doped $\mathrm{Zn}$ QDs degrade Methyl Violet (MV), the band gap for the doped QDs are increased due to the internal pressure of $\mathrm{Fe}^{3+}$ ions. The decolourization efficiency is decreases with increase in concentration owing to the occupancy of dye molecules at the surface of the catalyst that decreases the number of photons [31]. The photo catalyst behaves different manner in dissimilar medium and the bigger band gap enhances the photocatalytic activity [32]. The $\mathrm{Fe}^{3+}$ control the size of the progression of $\mathrm{ZnS}$ thus shows a blue shift in UV-Visible spectra. The reduction in sample absorbance than $\mathrm{ZnS}$ shows the importance of doping with $\mathrm{Fe}$ ions and it was examined by the decolourization of Victoria Blue R (VBR) with different mole fractions. The rate constant for decolourization of VBR decreases with increasing of concentration [33]. The band gap energy connects closely with the photocatalytic activity [34] and the dye molecules acts as the filter in greater amount causes the decrease in 
degradation efficiency. This can be prevented by fixing the optimum concentration and $8 \mathrm{mg}$ of capped ZnS QDs removes the VBR effectively [35]. Furthermore, ZnS QDs synthesized by chemical precipitation method using water as the solvent possess the particle size of $3 \mathrm{~nm}$ with zinc blende structure shows the most eminent photocatalytic activity against malachite green at $\mathrm{pH} 8$ and $0.80 \mathrm{~g} / \mathrm{L}$ within $2 \mathrm{hrs}$ [36]. CQDs/N-ZnO decolourizes malachite green, Methylene Blue and fluorescein contaminants to a greater extent under ordinary light of intensity $33 \times 103 \pm 50001 x$. Anti-photo corrosion property of CQDs increases the recycling tendency. Moreover, the band gap of $3.10 \mathrm{eV}$ depicts the broader absorption capacity [37].The GQDs modified with graphene increases the surface charges and surface area in water system induces high remotion, reusability and adsorption selectivity and high dispersion stability due to the electrostatic repulsion afforded by GQDs. This improved surface area break up the harmful coloured pollutants from water. The number of rings in the molecule decides the equilibrium adsorption performance irrespective of the nature of dye. In addition to that, Rhodamine B and Eriochrome Black can be desorbed from the QDs with the help of ethanol and ethylene glycol [38]. The CdSe QDs follows first order kinetics while degrading the coloured compounds such as basic violet 16, Basic Blue 41 and Acid Blue 92.The starch doped CdSe QDs photodecolourize $80 \%$ of malachite green and methylene green within three minutes in the ratio of 1:1000 catalyst and dye using MCR-ALS technique. This photo catalyst does not aggregate even after a long period of time due to the large number of hydroxyl group in complex carbohydrate [39]. The N-GQDs doped into $\mathrm{TiO}_{2}$ increases the decolourization performance of methylene blue to $85 \%$ due to the excitation and transfer of electrons from N-GQDs to $\mathrm{TiO}_{2}$ which forms oxygen and hydroxyl free radicals that oxidize the dye [40].

The GQD/TiO 2 nanotubes attained by hydrothermal method exhibits yellow fluorescence give rise to the size and functional groups at the surface. The enormous vacancy defects on the QDs leads to the excitation of wavelengths [41]. The raw chemical compound determines the particle size of the QDs. For instance, $\mathrm{SnO}_{2}$ QDs possess two different sizes with glutamic acid and aspartic acid due to the nature of the molecule present in amino acids [42].The impregnation of vertically aligned $\mathrm{TiO}_{2}$ with GQDs exhibits superior and highly stable photo decolourization of methylene blue even after numerous cycles [43].

\section{PESTICIDE}

It was proved that QDs can detect the pesticides even the existing concentration is below the regulatory limit. There is an advantage of doped QDs such as prolonged emission life time and less cytotoxicity over the undoped QDs [44]. The silica incorporated QDs are employed in creating fluorescence sensors to detect pesticides that, increase the chemical stability and decrease the photo bleaching effect [45]-[47]. The conjugation of QDs with macrocyclic molecules with cavities entails the pesticide detection.
Moreover calix[n]arene used as the receptors for pesticide selectively by changing the size of calix[n]arene. For instance, CdTe designed with p-sulfonatocalix [4] arene are employed for the detection of fenamithion and acetamiprid [48].

The incorporation of GO into NGQDs enhances the ElectroChemiluminescence (ECL) ability extremely with low onset potential. The behavior of ECL with $\mathrm{S}_{2} \mathrm{O}_{8}{ }^{2-}$ as a co reactant shows a strong ECL and the intensity is increases with increase in concentration of coreactant. The multipurpose chemical pentachlorophenol (PCP) is the very toxic pollutants. The permitted level in potable water is 9.85 $\mathrm{ng} / \mathrm{mL}$ [49], [50]. Graphene Oxide fabricated NGQDs exhibits excellent ECL in which graphene oxide (GO) behave as an immobilization platform and exaggerate the signal. The presence of Pentachlorophenol (PCP) decrease the ECL intensity through the oxidation into chloranil and the Relative Standard Deviation is (RSD) down to $2.2 \%$ at $\mathrm{pH} 8.0$ with limit of detection $0.03 \mathrm{pg} / \mathrm{mL}$ confirms its potent reproducibility [51]. When the magnetic silica beads/graphene quantum dots/molecularly imprinted polypyrrole (mSGP) capsulize with silica shows magnetic properties in addition with water dispersibility, selectivity and photoluminescence. mSGP is higly sensitive towards tributylin than mono and dibutylin. It is a potential composite to detect tributylin in sea water with limit of detection $42.56 \mathrm{ppb}$ [52]. The insecticide Oxamyl (N, Ndimethylcarbamoyloxyimino-2-(methylthio)acetamide)

enters the human body inhibits the function of human body enzyme. GQDs adsorb the maximum oxamyl at 250 rpm during agitation and this chemisorption follows pseudo second order kinetics. The removal efficiency increases with increase of temperature up to $40^{\circ} \mathrm{C}$ at $100 \mathrm{mg} / \mathrm{L}$ of oxamyl, on account of its strong interaction between the active site of the pesticide and QDs [53]. Moreover, the matrix effect affects the determination of organophosphorus pesticide (OPP) in low concentration level. This effect is suppressed by Magnetic Dispersive Solid Phase Micro Extraction (MDSPME) which separates the pesticide and pre concentrate out of actual sample in single step in which $\mathrm{Fe}$ reduced GQDs were used as a sorbent. One of the major advantages of this technique is takes place without any solvent. The extracting tendency is controlled by the type and volume of desorption solvent. Therefore, acetone is used as adsorption solvent owing to its desorption efficiency of 3.5 mins irrespective of the type of OPP. The rGOQDs possess very few polar functional groups at the surface enhances the extraction efficiency [54]. The environmental issues due to the use of diniconazole as fungicide and plant growth regulator can be detected using mMIP@CDs/QDs of which the low accessibility of binding site was resolved by MIP [55].

\section{PATHOGENS}

Microorganisms spreading through water are the causes of dreadful diseases like cholera, typhoid, Diarrhea. The detection of such pathogens is highly important in many fields of engineering sciences and molecular biology. The 
current detective technology needs modification or enrichment of the target pathogens in the sample. The unique properties of QDs such as broad absorption and narrow emission spectral profile, resistance to photo bleaching, larger stokes shift value, composition and size dependent absorption and emission, high molar extinction coefficient value and good quantum yield make it as an excellent material for detecting and identifying the microbes even in a tiny measures [56].For instance, the water soluble CdSe QDs excite the single wavelength, modify the optical system that measure the fluorescence and create a comfortable path to detect the pathogens [57]. This QDs exhibits extremely higher fluorescence intensity, luminosity and possess high stability than Fluorescein isothiocyanate and Tetramethyl Rhodamine Methyl Ester. Moreover, the binding of the above said QDs takes place at the top layer of the microorganisms [58]. Bio conjugated QDs open up a wider path in the exploitation of pathogenic detective system [59]. For the sensitive detection, the solid phase was formulated using the Immunuglobin $\mathrm{G}(\mathrm{IgG})$ polycarbonate membrane in addition with bio conjugated QDs. It was identified that the immunoassay for this system is extremely sensitive [60]. The three different colours are emanate from different QDs size. The ternary CdSe QDs/ graphene $/ \mathrm{TiO}_{2}$ composite exhibits photocatalytic antibacterial activity against E. coli under visible light, in which graphene/ $\mathrm{TiO}_{2}$ furnish support for the CdSe QDs. The photocatalytic activity against the bacteria damage the cell membrane and discharge the intercellular components [61]. The $\mathrm{TiO}_{2}$ nano tube increases the sensitivity of CdS QDs.

The GOQDs-Polyvinylidene Fluoride (PVDF) membrane exhibits more desirable bacteriocidal and antibiofouling properties were studied by testing E. coli bacteria of which the flux drop was $67.5 \%$ at $12 \mathrm{hrs}$ filtration. The spectral studies reveal the covalent bond formation between GOQDs and PVDF/The anti-biofouling property is decided by means of hydrophilicity which is evidenced by the flux drop of $24.3 \%$ [62].

The CQDs prepared from rice straw possess high light emitting capacity; sensitive and specific in bacterial surface and water solubility detect the pathogens effectively [63]. The duple properties photo catalytic and bactericide of $\mathrm{CQDs} / \mathrm{TiO}_{2}$ was investigated by comparing the properties with pristine $\mathrm{TiO}_{2}$. The pristine $\mathrm{TiO}_{2}$ acts as a photo catalyst upon irradiation with UV light. Whereas the fabricated QDs exhibits low recombination rate and operate as a photocatalyst under sun light that produce electron hole pairs [64].

\section{CONCLUSION}

In conclusion, the studies on QDs shown that GQDs, CQDs and inorganic QDs have significant effect on sewer water treatment. $\mathrm{Fe}^{3+}$ ion doped $\mathrm{ZnS}$ QDs remove $98 \%$ of the dyes from the polluted water effectively and N-GQDs doped $\mathrm{TiO}_{2}$ bleach the methylene blue efficaciously than the pristine $\mathrm{TiO}_{2}$. Additionally, $\mathrm{CQDs} / \mathrm{TiO}_{2}$ was the cost effective and efficient photocatalyst for the waste water treatment.

\section{REFERENCES}

[1] A. A. Alqadami, M. Naushad, M. A. Abdalla, M. R. Khan, Z. A Alothman, "Adsorptive Removal of Toxic Dye Using $\mathrm{Fe}_{3} \mathrm{O}_{4}-\mathrm{TSC}$ Nanocomposite: Equilibrium, Kinetic, and Thermodynamic Studies", J. Chem. Eng. Data, vol. 61, pp.3806-3813, Oct 2016.

[2] E. Daneshvar, A. Vazirzadeh, A. Niazi A et al, "Desorption of Methylene blue dye from brown macroalga: effects of operating parameters, isotherm study and kinetic modelling”, J Clean Prod, vol. 152, pp. 443-453, May 2017.

[3] M. Naushad, T. Ahamad, G. Sharma et al, "Synthesis and characterization of a new starch/SnO $\mathrm{O}_{2}$ nanocomposite for efficient adsorption of toxic $\mathrm{Hg}^{2+}$ metal ion", ChemEng J, vol. 300, pp. 306316.Sep 2016.

[4] G. Sharma, M. Naushad, D. Pathania, A. Kumar, "A multifunctional nano composite pectin thorium (IV) tungstomolybdate for heavy metal separation and photo remediation of malachite green", Desalin. Water Treat, vol.57, pp. 19443-19455, Sep 2016.

[5] Niyaz Mohammad Mahmoodi, Mokhtar Arami, "Numerical finite volume modeling of dye decolorization using immobilized titania nano photocatalysis", Chemical Engineering Journal, Vol. 146, pp 189-193, Feb 2009.

[6] F. D. Ardejani, K. H. Badii, N. L. Yousefi, N. M. Mahmoodi, M. Arami et al, "Numerical modelling and laboratory studies on the removal of Direct Red 23 and Direct Red 80 dyes from textile effluents using orange peel, a low-cost adsorbent", Dyes and Pigments, Vol. 73, pp. 178-185, Jan 2006.

[7] R. F. Barbieri, P. J. Lester, A.S. Miller, K.G. Ryan, "A neurotoxic pesticide changes the outcome of aggressive”, Proc. R. Soc. B Biol. Sci, vol. 280, pp. 2013-2157, Dec 2013.

[8] M. A. Beketov, B. J. Kefford, R. B. Schäfer, M. Liess, "Pesticides reduce regional biodiversity of stream invertebrates", Proc. Natl. Acad. Sci., vol. 110, pp. 11039-11043, U. S. A, 2013.

[9] G. Lautner, J. Kaev, J. Reut, A. Öpik, J. Rappich, V. Syritski, R. E. Gyurcsányi, "Selective Artificial Receptors Based on Micro patterned Surface-Imprinted Polymers for Label-Free Detection of Proteins by SPR Imaging”, Adv. Funct. Mater, vol. 21, pp. 21, 591597, Feb 2011.

[10] R. V. Shutov, A. Guerreiro, E. Moczko, I. P.de Vargas-Sansalvador, I. Chianella,, M.J.Whitcombe, S.A.Piletsky, "Introducing MINA-The Molecularly Imprinted Nanoparticle Assay”, Nano-micro Small, vol. 10, pp. 1086-1089, Mar 2014.

[11] A. P. Alivisatos, "Perspectives on the physical chemistry of semiconductor nanocrystals", J. Phys. Chem, vol. 100, pp. 1322613239, Aug 1996.

[12] D. Bera, L. Qian, P. H. Holloway, "Phosphor Quantum Dots", John Wiley \& Sons, Ltd; ch. 2, pp. 22, 2008, West Sussex, UK.

[13] A. D. Yoffe, Adv. Phys, "Low-dimensional systems: quantum size effects and electronic properties of semiconductor microcrystallites (zero-dimensional systems) and some quasi-two-dimensional systems", vol. 42, pp. 173-266, Jan 1993.

[14] S. C. Erwin, L. J. Zu, M. I. Haftel, A. L. Efros, T. A. Kennedy, D. J. Norris, "Doping semiconductor nanocrystals", Nature (London), vol.436, pp. 91-94, July 2005.

[15] D. J. Norris, A. L. Efros, S. C. Erwin, "Doped nanocrystals", Science, vol. 319, pp. 1776-1779, Mar 2008.

[16] H. S. Yang, S. Santra, P. H. Holloway, "Syntheses and applications of Mn-doped II-VI semiconductor nanocrystals", J. Nanosci. Nanotechnology, vol. 5, pp. 1364-1375, Sep 2005

[17] Y. A. Yang, O. Chen, A. Angerhofer, Y. C. Cao, "Radial-positioncontrolled doping in CdS/ZnS core/shell nanocrystals", J. Am. Chem. Soc, vol. 128, pp. 12428-12429, Sep 2006.

[18] Y. Wang, N. Herron, "Nanometer-sized semiconductor clusters: materials synthesis, quantum size effects, and photophysical properties", J. Phys. Chem, vol. 95, pp. 525-532, Jan 1991.

[19] X. Li, M. Rui, J. Song, Z. Shen and H. Zeng, "Carbon and graphene quantum dots for optoelectronic and energy devices: a review", Adv. Func. Mater, vol. 25, pp. 4929, July 2015.

[20] Y. Song, S. Zhu, S. Xiang, X. Zhao, J. Zhang, H. Zhang, Y. Fu, B. Yang, "Investigation into the fluorescence quenching behaviors and applications of carbon dots", Nanoscale, vol. 6, pp. 4676, Feb 2014.

[21] X. Hua, Y. Bao, Z. Chen, F. Wu, "Carbon quantum dots with intrinsic mitochondrial targeting ability for mitochondria-based theranostics", Nanoscale, vol. 9, pp. 10948, Jun 2017.

[22] Y. Wang Y, Hu A, "Carbon quantum dots: synthesis, properties and applications”, J. Mat. Chem, vol. 2, pp. 6921-6939, June 2014.

[23] SY. Lim, W. Shen, Z. Gao, "Carbon quantum dots and their applications", Chem. Soc. Rev, vol. 44, pp. 362-381, Oct 2014. 
[24] X. Zhou, Y. Zhang, C. Wang, X. Wu, Y. Yang, B. Zheng, H. Wu, S. Guo, J. Zhang, "Photo-Fenton reaction of graphene oxide: a new strategy to prepare graphene quantum dots for DNA cleavage", ACS Nano, vol.6,pp. 6592-6599, July 2012.

[25] Y. Dong, C. Chen, X. Zheng, L. Gao, Z. Cui, H. Yang, C. Guo, Y. Chi, C. M. Li, "One-step and high yield simultaneous preparation of single-and multi-layer graphene quantum dots from CX-72 carbon black", J. Mater. Chem, vol. 22, pp. 8764-8766, Mar 2012.

[26] V. Gupta, N. Chaudhary, R. Srivastava, G. D. Sharma, R. Bhardwaj, S. Chand, "Luminscent graphene quantum dots for organic photovoltaic devices", J. Am. Chem. Soc, vol.133, pp. 9960-9963, June 2011.

[27] Amandeep Kaur, Ahmad Umar, Sushil Kumar Kansal, "Sunlight driven photocatalytic degradation of non-steroidal antiinflammatory drug based on $\mathrm{TiO}_{2}$ quantum dots", Journal of Colloid and Interface Science, vol. 459, pp. 257-263, Dec 2015.

[28] G. Chayene, Anchieta, Daniela Sallet, L. Edson, Foletto, Syllos S.da Silva, Osvaldo Chiavone-Filho, Claudio A. O. do Nascimento, "Synthesis of ternary zinc spinel oxides and their application in the photo degradation of organic pollutant", Ceramics International, vol. 40, pp. 4173-4178, Apr 2014.

[29] Jun Zhang, Xueying Zhang, Shanshan Dong, Xian Zhou, Shuangshi Dong, " $\mathrm{N}$-doped carbon quantum dots/TiO $\mathrm{O}_{2}$ hybrid composites with enhanced visible light driven photocatalytic activity toward dye wastewater degradation and mechanism", Journal of Photochemistry and Photobiology A: Chemistry, vol. 325, pp. 104-110, July 2016.

[30] Gaurav Sharma, Anuj Kumar, Mu Naushad, Amit Kumar, Ala'a H. Al-Muhtaseb, Pooja Dhiman, Ayman A. Ghfar, Florian J. Stadler, M. R. Khand, "Photoremediation of toxic dye from aqueous environment using monometallic and bimetallic quantum dots based nanocomposites", Journal of Cleaner Production, vol. 172, pp. 29192930, June 2016.

[31] Mojtaba Shamsipur, Hamid Reza Rajabi, "Study ofphotocatalytic activity of ZnS quantum dots as efficient nanoparticles for removal of methyl violet: effect of ferric ion doping", Spectrochimica Acta Part A: Molecular and Biomolecular Spectroscopy, vol. 122, pp. 260-267, March 2014.

[32] M. J. Puchana-Rosero, Matthew A. Adebayo, Eder C. Lima Fernando M. Machado, Pascal S. T hue, Julio C. P. Vaghetti, Cibele S. Umpierres, Mariliz Gutterres, "Microwave-assisted activated carbon obtained from the sludge of tannery-treatment effluent plant for removal of leather dyes", Colloids and Surfaces A: Physicochemical and Engineering Aspects, Vol. 504, pp. 105-115, September 2016.

[33] Mojtaba Shamsipur, Hamid Reza Rajabi, Omid Khani, "Pure and $\mathrm{Fe}^{3+-}$ doped $\mathrm{ZnS}$ quantum dots as novel and efficient nano photocatalysts: Synthesis, characterization and use for decolorization of Victoria blue $R$ ", Materials Science in Semiconductor Processing,vol. 16, pp. 1154-1161, Aug 2013.

[34] Mohammad A. Behnajady, YasaminTohidi, "Synthesis, Characterization and Photocatalytic Activity of Mg-Impregnated $\mathrm{ZnO}-\mathrm{SnO}_{2}$ Coupled Nanoparticles", photochemistry and photobiology, vol. 90, pp. 51-56, Feb 2014.

[35] Hamid Reza Rajabi, HoomanArjmand, Hossein Kazemdehdashti, Mohammad Farsi, "Synthesis, Characterization and Photocatalytic Activity of Mg-Impregnated $\mathrm{ZnO}-\mathrm{SnO}_{2}$ Coupled Nanoparticles", Journal of Environmental Chemical Engineering, vol. 4, pp. 28302840, Sep 2016.

[36] Hamid Reza Rajabi, Omid Khani, Mojtaba Shamsipur, Vahid Vatanpour, "High-performance pure and $\mathrm{Fe}^{3+-i o n}$ doped $\mathrm{ZnS}$ quantum dots as green nanophotocatalysts for the removal of malachite green under UV-light irradiation", Journal of Hazardous Materials, vol. 250, pp. 370-378, Apr 2013.

[37] S. Muthulingam, In-Hwan Lee, Periyayya Uthirakumar, "Highly efficient degradation of dyes by carbon quantum dots/N-doped zinc oxide $(\mathrm{CQD} / \mathrm{N}-\mathrm{ZnO})$ photocatalyst and its compatibility on three different commercial dyes", Journal of Colloid and Interface Science, vol. 455, pp. 101-109, Oct 2015.

[38] Yulong Ying, Peng He, Guqiao Ding, Xinsheng Peng, "Ultrafast adsorption and selective desorption of aqueous aromatic dyes by graphene sheets modified by graphene quantum dots", Nanotechnology, vol. 27, Number 24, May 2016

[39] Bahram Hemmateenejad, ParisaShadabipour, TaherehKhosousi, MojtabaShamsipur, "Chemometrics investigation of the light-free degradation of methyl green and malachite green by starch-coated CdSe quantum dots", Journal of Industrial and Engineering Chemistry, vol. 27, pp. 384-390, July 2015.
[40] Hossein Safardoust-Hojaghan, Masoud Salavati-Niasari, "Degradation of methylene blue as a pollutant with $N$-doped graphene quantum dot/titanium dioxide nanocomposite", Journal of Cleaner Production, vol. 148, pp. 31-36, Apr 2017.

[41] Xu, Q. Zhou, Z. Hua, Q. Xue, C. Zhang, X. Wang, D. Pan, M. Xiao, "Single particle spectroscopic measurements of fluorescent graphene quantum dots", 'ACS nano, vol. 7, pp. 10654-10661, Nov 2013.

[42] Archita Bhattacharjee, M. Ahmaruzzaman, "A novel and green process for the production of tin oxide quantum dots and its application as a photocatalyst for the degradation of dyes from aqueous phase", Journal of Colloid and Interface Science, vol. 448, pp. 130-139, June 2015

[43] Bipin Kumar Gupta, GarimaKedawat, Yogyata Agrawal, Pawan Kumar, Jaya Dwivedia, S. K. Dhawana, "A novel strategy to enhance ultraviolet light driven photocatalysis from graphene quantum dots in filled $\mathrm{TiO}_{2}$ nanotube arrays", RSC Adv, vol. 5, pp. 10623-10631, Nov 2015.

[44] P. Wu, X.-P. Yan, “A Review of Methods for the Analysis of Orphan and Difficult Pesticides: Glyphosate, Glufosinate, Quaternary Ammonium and Phenoxy Acid Herbicides, and Dithiocarbamate and Phthalimide Fungicides", Chem. Soc. Rev, vol. 42, pp. 5489-5521, Mar 2013.

[45] H. Li, Y. Li, J. Cheng, "Imprinted photonic polymers for chiral recognition", Chem. Mater, vol. 22, pp. 2451-2457, Mar 2010.

[46] H. Li, F. Qu, "Synthesis of CdTe quantum dots in sol-gel-derived composite silica spheres coated with calix [4] arene as luminescent probes for pesticides", Chem. Mater, vol. 19, pp. 4148-4154, July 2007

[47] X.Hu, X.Gao, "Silica- Polymer Dual Layer-Encapsulated Quantum Dots with Remarkable Stability”, ACS Nano, vol. 4, pp. 6080-6086 Sep 2010.

[48] F.Qu, X. Zhou, J. Xu, H. Li, G. Xie, "Luminescence switching of CdTe quantum dots in presence of p-sulfonatocalix [4] arene to detect pesticides in aqueous solution", Talanta, vol. 78, pp. 13591363, Jun 2009

[49] W. Y. Shiu, K. C. Ma, D. Varhanickova and D. Mackay, "Chlorophenols and alkylphenols: a review and correlation of environmentally relevant properties and fate in an evaluative environment", Chemosphere, vol. 29, pp. 1155-1224, Sep 1994.

[50] L. H. Keith and W. A. Telliard, "ES\&T special report: priority pollutants: Ia perspective view", Environ. Sci. Technol, vol. 13, pp. 416-423, Apr 1979

[51] Xiaojiao Du, Ding Jiang, Qian Liu, Gangbing Zhu, Hanping Mao, Kun Wang, "Fabrication of graphene oxide decorated with nitrogendoped graphene quantum dots and its enhanced electrochemiluminescence for ultrasensitive detection of pentachlorophenol", Analyst, vol. 140, pp. 1253-1259, Apr 2015.

[52] Erhan Zor, Eden Morales-Narváez, Alejandro Zamora-Gálvez, Haluk Bingol, Mustafa Ersoz, ArbenMerkoçi, "Graphene quantum dotsbased photoluminescent sensor: a multifunctional composite for pesticide detection", ACS Appl. Mater. Interfaces, vol. 36, pp. 20272-20279, Aug 2015

[53] Shilpi Agarwal, Nima Sadeghi, Inderjeet Tyagi, Vinod Kumar Gupta, Ali Fakhri, "Adsorption of toxic carbamate pesticide oxamyl from liquid phase by newly synthesized and characterized graphene quantum dots nanomaterials", Journal of Colloid and Interface Science, vol. 478, pp. 430-438, Sep 2016.

[54] Samaneh Akbarzade, Mahmoud Chamsaz, Gholam Hossein Rounaghi, Mahdi Ghorbani, "Zero valent Fe-reduced graphene oxide quantum dots as a novel magnetic dispersive solid phase microextraction sorbent for extraction of organophosphorus", Analytical and Bioanalytical Chemistry, vol. 410, pp. 429-439, Apr 2018.

[55] Mohammad Amjadi, RoghayehJalili, "Molecularly imprinted mesoporous silica embedded with carbon dots and semiconductor quantum dots as a ratiometric fluorescent sensor for diniconazole, Biosensors and Bioelectronics", vol. 96, pp. 121-126, Oct 2017.

[56] Richa Jackeray, C. K. V. Zainul Abid, Gurpal Singh, Swati Jain, S. Chattopadhyaya, Sameer Sapra, T. G. Shrivastav, Harpal Singh, "Selective capturing and detection of Salmonella typhi on polycarbonate membrane using bio conjugated quantum dots", Talanta, vol. 84, pp. 952-962, May 2011.

[57] Jyoti K. Jaiswal, Hedi Mattoussi, J. Matthew Mauro \& Sanford M. Simon, "Long-term multiple color imaging of live cells using quantum dot bioconjugates", Nature Biotechnology, vol. 21, pp. 4751, May 2003.

[58] XiuhengXue, Jian Pan, HuimingXie, Juhua Wang, Shuang Zhang, "Fluorescence detection of total count of Escherichia coli and 
Staphylococcus aureus on water-soluble CdSe quantum dots coupled with bacteria", Talanta, vol. 77, pp. 1808-1813, Mar 2009.

[59] Pengju G. Luo, Fred J. Stutzenberger, "Nanotechnology in the detection and control of microorganisms", Advances in Applied Microbiology, vol.63, pp. 145-181, Jun 2008.

[60] Richa Jackeray, C. K. V. ZainulAbid, Gurpal Singh, Swati Jain, S. Chattopadhyaya, Sameer Sapra, T.G.Shrivastav, Harpal Singh, "Selective capturing and detection of Salmonella typhi on polycarbonate membrane using bioconjugated quantum dots", Talanta, vol. 84, pp. 952-962, May 2011.

[61] Xiyang Ma, Quanjun Xiang, Yulong Liao, Tianlong Wen, Huaiwu Zhang, "Visible-light-driven CdSe quantum dots/graphene/TiO $\mathrm{O}_{2}$ nanosheets composite with excellent photocatalytic activity for E. coli disinfection and organic pollutant", Applied Surface Science, vol. 457, pp. 846-855,Nov 2018.

[62] Zhiping Zeng, Dingshan Yu, Ziming He, Jing Liu, Fang-Xing Xiao, Yan Zhang, Rong Wang, Dibakar Bhattacharyya, Timothy Thatt Yang Tan, "Graphene oxide quantum dots covalently functionalized PVDF membrane with significantly-enhanced bactericidal and antibiofouling performances", Scientific Reports, vol. 6, Article number: 20142, Feb 2016.

[63] K. Mandal, Tapas, Parvin, Nargish, "Rapid detection of bacteria by carbon quantum dots", Journal of Biomedical Nanotechnology, vol. 7, pp. 846-848, Dec 2011.

[64] Prem Singh Saud, Bishweshwar Pant, Al-MahmnurAlam, Zafar Khan Ghouri, Mira Park, Hak-Yong Kim, "Carbon quantum dots anchored $\mathrm{TiO}_{2}$ nanofibers: Effective photocatalyst for waste water treatment", Ceramics International, vol. 41, Part B, pp. 11953 11959, Nov 2015

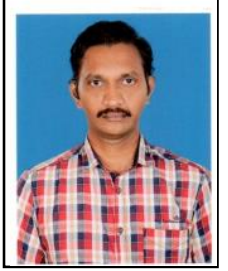

Dr. K. V. Anand pursued his Ph. D in the year 2014 from Bharathidasan University, Tiruchirappalli, Tamilnadu, India. He is currently working as a Lecturer in the Institute of Virtual and Distance Learning (IVDL) under DMI St. Eugene University, Lusaka, Zambia. To his credit he has published research papers in reputed international journals. His main research work focuses on stress biology. He has 6 years of teaching and research experience.

Dr. A. Sandy Subala pursued her Ph. D in the year 2012 from Acharya Nagarjuna University, Guntur, Andhra Pradesh, India. She is currently working as a senior Lecturer in the Institute of Virtual and Distance Learning (IVDL) under DMI St. Eugene University, Lusaka, Zambia. To her credit she has published research papers in reputed international journals. Her main research work focuses on Liquid crystal. She has 6 years of teaching and research experience.

Dr. Sumathi K. Sripathi is a doctorate from Madurai Kamara University, Madurai, on a multidisciplinary concept of academic study. She is at present working as a Senior Lecturer in the Institute of Virtual and Distance Learning of the DMI St. Eugene University, Lusaka, Zambia teaching Biology and allied applications. She has published research papers in multidisciplinary percepts in reputed international journals. Her research work focuses on sustainable development and womanism. She has 37 years of teaching experience with 5 years of research evaluation.

Ms. S. Antony Lucia Merin is working as an Assistant Lecturer in DMI - St. Eugene University, Zambia. She has completed Bachelor of Engineering in the field of Computer Science in Engineering (CSE) at St. Xavier's Catholic College of Engineering, Anna University, Chennai, India Her Master of Engineering in the field of (CSE - Networking) at Rohini College of Engineering and Technology, Anna University, Chennai India. 